\title{
EXISTENCE AND GLOBAL STABILITY OF PERIODIC SOLUTION FOR DELAYED DISCRETE HIGH-ORDER HOPFIELD-TYPE NEURAL NETWORKS
}

\author{
HONG XIANG, KE-MING YAN, AND BAI-YAN WANG
}

Received 6 February 2005

By using coincidence degree theory as well as a priori estimates and Lyapunov functional, we study the existence and global stability of periodic solution for discrete delayed highorder Hopfield-type neural networks. We obtain some easily verifiable sufficient conditions to ensure that there exists a unique periodic solution, and all theirs solutions converge to such a periodic solution.

\section{Introduction}

It is well known that studies on neural dynamical systems not only involve discussion of stability property, but also involve other dynamics behaviors such as periodic oscillatory, bifurcation and chaos. In many applications, the property of periodic oscillatory solutions are of great interest. For example, the human brain has been in periodic oscillatory or chaos state, hence it is of prime importance to study periodic oscillatory and chaos phenomenon of neural networks. Recently, Liu and Liao [8], Zhou and Liu [15] consider the existence and global exponential stability of periodic solutions of delayed Hopfield neural networks and delayed cellular neural networks. Liu et al. [7] address the existence and global exponential stability of periodic solutions of delayed BAM neural networks. Since high-order neural networks have stronger approximation property, faster convergence rate, greater storage capacity, and higher fault tolerance than lower-order neural networks, they have attracted considerable attention (see, e.g., $[1,2,4,5,10,11,13,14]$ ). In our previous paper [12], we study the global exponential stability and existence of periodic solutions of the following high-order Hopfield-type neural networks

$$
\begin{aligned}
\frac{d x_{i}(t)}{d t}= & -a_{i}(t) x_{i}(t)+\sum_{j=1}^{m} b_{i j}(t) f_{j}\left(x_{j}(t)\right) \\
& +\sum_{j=1}^{m} \tilde{b}_{i j}(t) f_{j}\left(x_{j}\left(t-\tau_{i j}(t)\right)\right)+\sum_{j=1}^{m} \sum_{l=1}^{m} e_{i j l}(t) f_{j}\left(x_{j}(t)\right) f_{l}\left(x_{l}(t)\right) \\
& +\sum_{j=1}^{m} \sum_{l=1}^{m} \tilde{e}_{i j l}(t) f_{j}\left(x_{j}\left(t-\sigma_{i j l}(t)\right)\right) f_{l}\left(x_{l}\left(t-\sigma_{i j l}(t)\right)\right)+I_{i}(t),
\end{aligned}
$$


where $i=1,2, \ldots, m, t>0, x_{i}(t)$ denotes the potential (or voltage) of the cell $i$ at time $t$. $a_{i}(t)$ are positive $\omega$-periodic functions, they denote the rate with which the cell $i$ reset their potential to the resting state when isolated from the other cells and inputs. $b_{i j}(t), e_{i j l}(t)$ are the first-and second-order connection weights of the neural network, respectively; $I_{i}(t)$ denote the $i$ th component of an external input source introduced from outside the network to the cell $i$.

In [6], Li investigates global stability and existence of periodic solutions of discrete delayed cellular neural networks. However, few authors have studies the dynamical behaviors of the discrete-time analogues of delayed high-order Hopfield-type neural networks with variable coefficient. In this paper, we are concerned with the following discrete analogue of (1.1) of the form

$$
\begin{aligned}
x_{i}(n+1)= & e^{-a_{i}(n) h} x_{i}(n)+\theta_{i}(h) \sum_{j=1}^{n} b_{i j}(n) f_{j}\left(x_{j}(n)\right) \\
& +\theta_{i}(h) \sum_{j=1}^{n} \widetilde{b}_{i j}(n) f_{j}\left(x_{j}\left(n-\tau_{i j}(n)\right)\right)+\theta_{i}(h) \sum_{j=1}^{n} \sum_{l=1}^{n} e_{i j l}(n) f_{j}\left(x_{j}(n)\right) f_{l}\left(x_{l}(n)\right) \\
& +\theta_{i}(h) \sum_{j=1}^{n} \sum_{l=1}^{n} \widetilde{e}_{i j l}(n) f_{j}\left(x_{j}\left(n-\sigma_{i j l}(n)\right)\right) f_{l}\left(x_{l}\left(t-\sigma_{i j l}(n)\right)\right) \\
& +\theta_{i}(h) I_{i}(n), \quad i=1,2, \ldots, m,
\end{aligned}
$$

in which $\theta_{i}, a_{i}, b_{i j}, e_{i j l}, i, j, l=1,2, \ldots, m$, will be specified in the next section.

With the help of Mawhin's continuation theorem of coincidence degree theory and constructing Lyapunov functional, we obtain some sufficient conditions ensure that for the discrete networks (1.2) there exists a unique periodic solution, and all theirs solutions converge to such a periodic solution. To the best of our knowledge, this is the first time to study the existence and global attractivity of the periodic solution for the discrete-time analogues of delayed high-order Hopfield-type neural networks with variable coefficient.

The tree of this paper is as follows. In Section 2, following the semi-discretization technique $[6,9]$, we obtain a discrete-time analogue of (1.1). In Section 3, with the help of Mawhin's continuation theorem of coincidence degree theory, we study the existence of the periodic solution of (1.2). In Section 4, by constructing Lyapunov functional, we derive sufficient conditions to ensure that the periodic solution of (1.2) is globally asymptotically stable.

\section{Discrete-time analogues}

There is no unique way of deriving discrete time version of dynamical equations corresponding to continuous time formulation. First, following [6, 9], we reformulate system (1.1) by an approximation of the form

$$
\begin{aligned}
\frac{d x_{i}(t)}{d t}= & -a_{i}\left(\left[\frac{t}{h}\right] h\right) x_{i}(t)+\sum_{j=1}^{m} b_{i j}\left(\left[\frac{t}{h}\right] h\right) f_{j}\left(x_{j}\left(\left[\frac{t}{h}\right] h\right)\right) \\
& +\sum_{j=1}^{m} \tilde{b}_{i j}\left(\left[\frac{t}{h}\right] h\right) f_{j}\left(x_{j}\left(\left(\left[\frac{t}{h}\right] h\right)-\tau_{i j}\left(\left[\frac{t}{h}\right] h\right)\right)\right)
\end{aligned}
$$




$$
\begin{aligned}
& +\sum_{j=1}^{m} \sum_{l=1}^{m} e_{i j l}\left(\left[\frac{t}{h}\right] h\right) f_{j}\left(x_{j}\left(\left[\frac{t}{h}\right] h\right)\right) f_{l}\left(x_{l}\left(\left[\frac{t}{h}\right] h\right)\right) \\
& +\sum_{j=1}^{m} \sum_{l=1}^{m} \tilde{e}_{i j l}\left(\left[\frac{t}{h}\right] h\right) f_{j}\left(x_{j}\left(\left(\left[\frac{t}{h}\right] h\right)-\sigma_{i j l}\left(\left[\frac{t}{h}\right] h\right)\right)\right) \\
& \times f_{l}\left(x_{l}\left(\left(\left[\frac{t}{h}\right] h\right)-\sigma_{i j l}\left(\left[\frac{t}{h}\right] h\right)\right)\right)+I_{i}\left(\left[\frac{t}{h}\right] h\right),
\end{aligned}
$$

where $h$ is a positive number denoting a uniform discretization step size and $[t / h]$ denotes the greatest integer in $t / h$. For convenience, we denote $[t / h]=n, n \in Z_{0}^{+}$, and $a_{i}(n h)=$ $a_{i}(n), b_{i j}(n h)=b_{i j}(n), \widetilde{b}_{i j}(n h)=\tilde{b}_{i j}(n), e_{i j l}(n h)=e_{i j l}(n), \widetilde{e}_{i j l}(n h)=\widetilde{e}_{i j l}(n), \tau_{i j}(n h)=\tau_{i j}$, $\sigma_{i j l}(n h)=\sigma_{i j l}, x_{i}(n h)=x_{i}(n), I_{i}(n h)=I_{i}$. Thus (2.1) takes on the form

$$
\begin{aligned}
\frac{d x_{i}(t)}{d t}= & -a_{i}(n) x_{i}(t)+\sum_{j=1}^{m} b_{i j}(n) f_{j}\left(x_{j}(n)\right) \\
& +\sum_{j=1}^{m} \tilde{b}_{i j}(n) f_{j}\left(x_{j}\left(n-\tau_{i j}(n)\right)\right) \\
& +\sum_{j=1}^{m} \sum_{l=1}^{m} e_{i j l}(n) f_{j}\left(x_{j}(n)\right) f_{l}\left(x_{l}(n)\right) \\
& +\sum_{j=1}^{m} \sum_{l=1}^{m} \tilde{e}_{i j l}(n) f_{j}\left(x_{j}\left(n-\sigma_{i j l}(n)\right)\right) \\
& \times f_{l}\left(x_{l}\left(n-\sigma_{i j l}(n)\right)\right)+I_{i}(n), \quad n \in Z_{0}^{+} .
\end{aligned}
$$

Integrate it over the interval $[n h, t]$ for $t<(n+1) h$ to obtain

$$
\begin{aligned}
& x_{i}(t) e^{a_{i}(n) t}-x_{i}(n) e^{a_{i}(n) n h} \\
&=\frac{e^{a_{i}(n) t}-e^{a_{i}(n) n h}}{a_{i}(n)}\left\{\sum_{j=1}^{m} b_{i j}(n) f_{j}\left(x_{j}(n)\right)+\sum_{j=1}^{m} \tilde{b}_{i j}(n) f_{j}\left(x_{j}\left(n-\tau_{i j}(n)\right)\right)\right. \\
&+\sum_{j=1}^{m} \sum_{l=1}^{m} e_{i j l}(n) f_{j}\left(x_{j}(n)\right) f_{l}\left(x_{l}(n)\right) \\
&+\sum_{j=1}^{m} \sum_{l=1}^{m} \tilde{e}_{i j l}(n) f_{j}\left(x_{j}(n)\right) f_{l}\left(x_{l}(n)\right) f_{l}\left(x_{l}\left(n-\sigma_{i j l}(n)\right)\right) \\
&\left.+I_{i}(n)\right\}, \quad i=1,2, \ldots, m .
\end{aligned}
$$


We let $t \rightarrow(n+1) h$ and obtain

$$
\begin{aligned}
x_{i}(n+1)= & x_{i}(n) e^{-a_{i}(n) h}+\theta_{i}(h) \sum_{j=1}^{m} b_{i j}(n) f_{j}\left(x_{j}(n)\right) \\
& +\theta_{i}(h) \sum_{j=1}^{m} \tilde{b}_{i j}(n) f_{j}\left(x_{j}\left(n-\tau_{i j}(n)\right)\right) \\
& +\theta_{i}(h) \sum_{j=1}^{m} \sum_{l=1}^{m} e_{i j l}(n) f_{j}\left(x_{j}(n)\right) f_{l}\left(x_{l}(n)\right) \\
& +\theta_{i}(h) \sum_{j=1}^{m} \sum_{l=1}^{m} \tilde{e}_{i j l}(n) f_{j}\left(x_{j}\left(n-\sigma_{i j l}(n)\right)\right) f_{l}\left(x_{l}\left(n-\sigma_{i j l}(n)\right)\right) \\
& +\theta_{i}(h) I_{i}(n), \quad i=1,2, \ldots, m, n \in Z_{0}^{+},
\end{aligned}
$$

where

$$
\theta_{i}(h)=\frac{1-e^{-a_{i}(n) h}}{a_{i}(n)}, \quad i=1,2, \ldots, m, n \in Z_{0}^{+} .
$$

It is not difficult to verify that $\theta_{i}(h)>0$ if $a_{i}, h>0$ and $\theta_{i}(h) \approx h+o\left(h^{2}\right)$ for small $h>0$. Also, one can see that (1.2) converges towards (1.1) when $h \rightarrow 0^{+}$. The system (1.2) is supplemented with initial values given by

$$
x_{i}(s)=\varphi_{i}(s), \quad s \in\left(-\tau^{*}, 0\right)_{Z}, \tau^{*}=\max _{1 \leq i, j, l \leq m}\left(\max _{n \in Z}\left(\tau_{i j}(n), \sigma_{i j l}(n)\right)\right) .
$$

In this paper, we assume that

$(H 1) a_{i}: Z \rightarrow(0, \infty), b_{i j}, \tilde{b}_{i j}, e_{i j l}, \tilde{e}_{i j l}, I_{i} \in Z \rightarrow R, \tau_{i j}, \sigma_{i j l}: Z \rightarrow Z_{0}^{+}, i, j, l=1,2, \ldots, m$, $h \in(0, \infty)$.

(H2) $f_{j}$ are Lipschitzian with Lipschitz constants $L_{j}>0$,

$$
\left|f_{j}(x)-f_{j}(y)\right| \leq L_{j}|x-y|
$$

for any $x, y \in R,(j \in\{1, \ldots, m\})$.

(H3) There exist positive constants $N_{j}>0, j \in\{1, \ldots, m\}$ such that

$$
\left|f_{j}(x)\right| \leq N_{j}, \quad j \in\{1, \ldots, m\} .
$$

For convenience, we will introduce the notation:

$$
I_{\omega}=\{0,1, \ldots, \omega-1\}, \quad \bar{u}=\frac{1}{\omega} \sum_{k=0}^{\omega-1} u(k),
$$


where $u(k)$ an $\omega$-perodic sequence of real numbers defined for $k \in Z$ and notations:

$$
\begin{array}{cc}
\underline{a}_{i}=\min _{n \in I_{\omega}}\left(a_{i}(n)\right), & i=1,2, \ldots, m, \\
I^{M}=\max _{n \in I_{\omega}}\left\{\left|I_{i}(n)\right|, i=1,2, \ldots, m\right\}, & M=\sup _{u \in R}\left\{\left|f_{j}(u)\right|, j=1,2, \ldots, m\right\}, \\
b_{i j}^{M}=\max _{n \in I_{\omega}}\left\{\left|b_{i j}(n)\right|\right\}, & \tilde{b}_{i j}^{M}=\max _{n \in I_{\omega}}\left\{\left|\tilde{b}_{i j}(n)\right|\right\} \\
e_{i j l}^{M}=\max _{n \in I_{\omega}}\left\{\left|e_{i j l}(n)\right|\right\}, & \tilde{e}_{i j l}^{M}=\max _{n \in I_{\omega}}\left\{\left|\tilde{e}_{i j l}(n)\right|\right\} .
\end{array}
$$

\section{Existence of periodic solution}

In this section, based on the Mawhin's continuation theorem and Lyapunov functional, we will study the existence of periodic solutions of discrete-time high-order Hopfieldtype neural networks (1.2).

First, we will make some preparations.

Let $X$ and $Z$ be two Banach spaces. Consider an operator equation

$$
L x=\lambda N x, \quad \lambda \in(0,1),
$$

where $L: \operatorname{Dom} L \cap X \rightarrow Z$ is a linear operator and $\lambda$ is a parameter. Let $P$ and $Q$ denote two projectors such that

$$
P: X \cap \operatorname{Dom} L \longrightarrow \operatorname{Ker} L, \quad Q: Z \longrightarrow \frac{Z}{\operatorname{Im}} L
$$

Denote by $H: \operatorname{Im} Q \rightarrow \operatorname{Ker} L$ is an isomorphism of $\operatorname{Im} Q$ onto $\operatorname{Ker} L$. In the sequel, we will use the following result of Mawhin [3, page 40].

Lemma 3.1. Let $X$ and $Z$ be two Banach spaces and $L$ a Fredholm mapping of index zero. Assume that $N: \bar{\Omega} \rightarrow Z$ is L-compact on $\bar{\Omega}$ with $\Omega$ open bounded in X. Furthermore assume:

(a) for each $\lambda \in(0,1), y \in \partial \Omega \cap \operatorname{Dom} L$,

$$
L x \neq \lambda N x
$$

(b) for each $x \in \partial \Omega \cap \operatorname{Ker} L$,

$$
Q N x \neq 0, \quad \operatorname{deg}\{H Q N x, \Omega \cap \operatorname{Ker} L, 0\} \neq 0 .
$$

Then the equation $L x=N x$ has at least one solution in $\operatorname{dom} L \cap \bar{\Omega}$.

Recall that a linear mapping $L: \operatorname{Dom} L \cap x \rightarrow Z$ with $\operatorname{Ker} L=L^{-1}(0)$ and $\operatorname{Im} L=$ $L(\operatorname{Dom} L)$, will be called a Fredholm mapping if the following two conditions hold:

(i) $\operatorname{Ker} L$ has a finite dimension;

(ii) $\operatorname{Im} L$ is closed and has a finite codimension. 
Recall also that the codimension of $\operatorname{Im} L$ is the dimension of $Z / \operatorname{Im} L$, that is, the dimension of the cokernel coker $L$ of $L$.

When $L$ is a Fredholm mapping, its index is the integer $\operatorname{Ind} L=\operatorname{dim} \operatorname{ker} L$-codim $\operatorname{Im} L$.

We will say that a mapping $N$ is $L$-compact on $\Omega$ if the mapping $Q N: \bar{\Omega} \rightarrow Z$ is continuous, $Q N(\bar{\Omega})$ is bounded, and $K_{p}(I-Q) N: \bar{\Omega} \rightarrow Y$ is compact, that is, it is continuous and $K_{p}(I-Q) N(\bar{\Omega})$ is relatively compact, where $K_{p}: \operatorname{Im} L \rightarrow \operatorname{Dom} L \cap \operatorname{Ker} P$ is a inverse of the restriction $L_{p}$ of $L$ to $\operatorname{Dom} L \cap \operatorname{Ker} P$, so that $L K_{p}=I$ and $K_{p} L=I-P$.

Next, we will state and prove the existence of periodic solutions of system (1.2).

Theorem 3.2. Assume that the condition (H1), (H2), and (H3) are satisfied. Furthermore, assume that

(H4) $a_{i}, b_{i j}, \tilde{b}_{i j}, e_{i j l}, \tilde{e}_{i j l}, I_{i}, i, j, l=1,2, \ldots, m$, are all $\omega$-periodic functions.

Then system (1.2) has at least one $\omega$-periodic solution.

Proof. Similar to that of [6], we define

$$
l_{m}=\left\{x=\{x(k)\}: x(k) \in R^{m}, k \in Z\right\}
$$

Let $l^{\omega} \subset l_{m}$ denote the subspace of all $\omega$ periodic sequences equipped with the usual supremum norm $\|\cdot\|$, that is,

$$
\begin{aligned}
\|x\| & =\left\|\left(x_{1}(k), \ldots, x_{m}(k)\right)^{T}\right\| \\
& =\sum_{i=1}^{m} \max _{k \in I_{\omega}}\left|x_{i}(k)\right|, \quad \text { for any } x=\left\{\left(x_{1}(k), \ldots, x_{m}(k)\right): k \in Z\right\} \in l^{\omega} .
\end{aligned}
$$

It is not difficult to show that $l^{\omega}$ is a finite-dimensional Banach space.

Let

$$
\begin{aligned}
& l_{0}^{\omega}=\left\{x=\{x(k)\} \in l^{\omega}: \sum_{k=0}^{\omega-1} x(k)=0\right\}, \\
& l_{c}^{\omega}=\left\{x=\{x(k)\} \in l^{\omega}: x(k)=h \in R^{m}, k \in Z\right\},
\end{aligned}
$$

then it follows that $l_{0}^{\omega}$ and $l_{c}^{\omega}$ are both closed linear subspaces of $l^{\omega}$ and

$$
l^{\omega}=l_{0}^{\omega} \oplus l_{c}^{\omega}, \quad \operatorname{dim} l_{c}^{\omega}=m
$$


In order to use Lemma 3.1 to system (1.2), we take $X=Y=l^{\omega},(L x)(k)=x(k+1)-x(k)$, and let

$$
\begin{aligned}
N x(n)=\left[\begin{array}{c}
x_{1}(n)\left(e^{-a_{1}(n) h}-1\right)+\theta_{1}(h) \sum_{j=1}^{m} b_{1 j}(n) f_{j}\left(x_{j}(n)\right)+ \\
\vdots \\
x_{m}(n)\left(e^{-a_{m}(n) h}-1\right)+\theta_{m}(h) \sum_{j=1}^{m} b_{m j}(n) f_{j}\left(x_{j}(n)\right)+
\end{array}\right. \\
\quad \theta_{1}(h) \sum_{j=1}^{m} \tilde{b}_{1 j}(n) f_{j}\left(x_{j}\left(n-\tau_{1 j}(n)\right)\right)+\theta_{1}(h) I_{1}(n) \\
\quad \begin{array}{c}
\theta_{m}(h) \sum_{j=1}^{m} \tilde{b}_{m j}(n) f_{j}\left(x_{j}\left(n-\tau_{m j}(n)\right)\right)+\theta_{m}(h) I_{m}(n) \\
+
\end{array} \\
+\theta_{1}(h) \sum_{j=1}^{m} \sum_{l=1}^{m} e_{1 j l}(n) f_{j}\left(x_{j}(n)\right) f_{l}\left(x_{l}(n)\right)+ \\
\quad \vdots \\
\quad \theta_{1}(h) \sum_{j=1}^{n} \sum_{l=1}^{n} \sum_{l=1}^{n} \tilde{e}_{1 j l}(n) f_{j}\left(x_{j}\left(n-\sigma_{1 j l}(n)\right)\right) f_{l}\left(x_{l}\left(t-\sigma_{1 j l}(n)\right)\right) \\
\vdots \\
j=1
\end{aligned}
$$

It is trivial to see that $L$ is a bounded linear operator and

$$
\operatorname{Ker} L=l_{c}^{\omega}, \quad \operatorname{Im} L=l_{0}^{\omega},
$$

as well as

$$
\operatorname{dim} \operatorname{Ker} L=m=\operatorname{codim} \operatorname{Im} L
$$

then it follows that $L$ is a Fredholm mapping of index zero.

Define

$$
P x=\frac{1}{\omega} \sum_{s=0}^{\omega-1} x(s), \quad x \in X, \quad Q z=\frac{1}{\omega} \sum_{s=0}^{\omega-1} z(s), \quad z \in Y .
$$

It is not difficult to show that $P$ and $Q$ are continuous projectors such that

$$
\operatorname{Im} P=\operatorname{Ker} L, \quad \operatorname{Im} L=\operatorname{Ker} Q=\operatorname{Im}(I-Q) .
$$


Furthermore, the generalized inverse (to $L) K_{p}: \operatorname{Im} L \rightarrow \operatorname{Ker} P \cap \operatorname{dom} L$ has the form

$$
K_{p}(z)=\sum_{s=0}^{k-1} z(s)-\frac{1}{\omega} \sum_{s=0}^{\omega-1}(\omega-s) z(s) .
$$

Clearly, $Q N$ and $K_{p}(I-Q) N$ are continuous. Since $X$ is a finite-dimensional Banach space, using the Arzela-Ascoli theorem, it is not difficult to show that $Q N(\bar{\Omega}), K_{p}(I-$ Q) $N(\bar{\Omega})$ are relatively compact for any open bounded set $\Omega \subset X$. Hence, $N$ is $L$-compact on $\bar{\Omega}$, here $\Omega$ is any open bounded set in $X$.

Now we reach the position to search for an appropriate open, bounded subset $\Omega$ for the application of the Lemma 3.1. Corresponding to the operator equation $L x=\lambda N x$, $\lambda \in(0,1)$, we have

$$
\begin{aligned}
x_{i}(n+1)-x_{i}(n)=\lambda( & x_{i}(n)\left(e^{-a_{i}(n) h}-1\right)+\theta_{i}(h) \sum_{j=1}^{m} b_{i j}(n) f_{j}\left(x_{j}(n)\right) \\
& +\theta_{i}(h) \sum_{j=1}^{m} \tilde{b}_{i j}(n) f_{j}\left(x_{j}\left(n-\tau_{i j}(n)\right)\right) \\
& +\theta_{i}(h) \sum_{j=1}^{m} \sum_{l=1}^{m} e_{i j l}(n) f_{j}\left(x_{j}(n)\right) f_{l}\left(x_{l}(n)\right) \\
& +\theta_{i}(h) \sum_{j=1}^{m} \sum_{l=1}^{m} \tilde{e}_{i j l}(n) f_{j}\left(x_{j}\left(n-\sigma_{i j l}(n)\right)\right) f_{l}\left(x_{l}\left(n-\sigma_{i j l}(n)\right)\right) \\
& \left.+\theta_{i}(h) I_{i}(n)\right), \quad i=1,2, \ldots, m .
\end{aligned}
$$

Assume that $x(n)=\left(x_{1}(n), \ldots, x_{m}(n)\right) \in X$ is a solution of system (3.15) for some $\lambda \in$ $(0,1)$, from $(3.15)$, we obtain

$$
\begin{aligned}
\max _{n \in I_{\omega}}\left|x_{i}(n)\right|= & \max _{n \in I_{\omega}}\left|x_{i}(n+1)\right| \\
\leq & \left(1+\lambda\left(e^{-a_{i}(n) h}-1\right)\left|x_{i}(n)\right|+\lambda \theta_{i}(h) \sum_{j=1}^{m}\left|b_{i j}(n)\right|\left|f_{j}\left(x_{j}(n)\right)\right|\right. \\
& +\lambda \theta_{i}(h) \sum_{j=1}^{m} \tilde{b}_{i j}(n) f_{j}\left(x_{j}\left(n-\tau_{i j}(n)\right)\right) \\
& +\lambda \theta_{i}(h) \sum_{j=1}^{m} \sum_{l=1}^{m} e_{i j l}(n) f_{j}\left(x_{j}(n)\right) f_{l}\left(x_{l}(n)\right) \\
& \left.+\lambda \theta_{i}(h) \sum_{j=1}^{m} \sum_{l=1}^{m} \tilde{e}_{i j l}(n) f_{j}\left(x_{j}\left(n-\sigma_{i j l}(n)\right)\right) f_{l}\left(x_{l}\left(n-\sigma_{i j l}(n)\right)\right)+\lambda \theta_{i}(h) I_{i}(n)\right)
\end{aligned}
$$




$$
\begin{aligned}
\leq & \left(1+\lambda\left(e^{-\underline{a}_{i}(n) h}-1\right)\right) \max _{n \in I_{\omega}}\left|x_{i}(n)\right|+\lambda \theta_{i}(h) m b M+\lambda \theta_{i}(h) m \tilde{b} M \\
& +\lambda \theta_{i}(h) m^{2} e M^{2}+\lambda \theta_{i}(h) m^{2} \tilde{e} M^{2}+\lambda \theta_{i}(h) I^{M}, \quad i=1,2, \ldots, m .
\end{aligned}
$$

Hence,

$$
\begin{aligned}
& \left(1-e^{-\underline{a}_{i}(n) h}\right) \max _{n \in I_{\omega}}\left|x_{i}(n)\right| \\
& \quad \leq \theta_{i}(h)\left(m b M+m \tilde{b} M+m^{2} e M^{2}+m^{2} \widetilde{e} M^{2}+I^{M}\right), \quad i=1,2, \ldots, m .
\end{aligned}
$$

That is

$$
\max _{n \in I_{\omega}}\left|x_{i}(n)\right| \leq \frac{\theta_{i}(h)\left(m b M+m \tilde{b} M+m^{2} e M^{2}+m^{2} \tilde{e} M^{2}+I^{M}\right)}{1-e^{-a_{i}}(n) h}:=A_{i} .
$$

Denote $A=\sum_{i=1}^{m} A_{i}+E$, where $E$ is taken sufficiently large such that $\|x\|<A$, clearly, $A$ is independent of $\lambda$. Now, we take $\Omega=\{u \in X:\|x\|<A\}$. It is clear that $\Omega$ satisfies the requirement $(a)$ in Lemma 3.1 .

When $x \in \partial \Omega \cap \operatorname{Ker} L, x$ is a constant vector in $R^{m}$ with $\|x\|=A$. Furthermore, take $H: \operatorname{Im} Q \rightarrow \operatorname{Ker} L, r \rightarrow r$. we can let $A$ be greater such that

$$
\begin{aligned}
& \left(x_{1}, \ldots, x_{m}\right) \text { HQN }\left(\begin{array}{c}
x_{1} \\
\vdots \\
x_{m}
\end{array}\right) \\
& =\sum_{i=1}^{n}\left(-x_{i}^{2} \sum_{s=0}^{\omega-1}\left(\frac{e^{-a_{i}(s) h}-1}{\omega}\right)+\theta_{i}(h) x_{i} \sum_{j=1}^{m} \bar{b}_{i j} f_{j}\left(x_{j}\right) x_{i}\right. \\
& +\theta_{i}(h) x_{i} \sum_{j=1}^{m} \overline{\widetilde{b}}_{i j} f_{j}\left(x_{j}\right) x_{i}+\theta_{i}(h) \sum_{j=1}^{m} \sum_{l=1}^{m} \bar{e}_{i j l} f_{j}\left(x_{j}\right) f_{l}\left(x_{l}\right) x_{i} \\
& \left.+\theta_{i}(h) \sum_{j=1}^{m} \sum_{l=1}^{m} \overline{\widetilde{e}}_{i j l} f_{j}\left(x_{j}\right) f_{l}\left(x_{l}\right) x_{i}+\theta_{i}(h) \bar{I}_{i} x_{i}\right)<0 .
\end{aligned}
$$

So for any $x \in \partial \Omega \cap \operatorname{Ker} L, Q N x \neq 0$. Furthermore, let $\Psi(r ; u)=-r x+(1-r) J Q N x$, then for any $x \in \partial \Omega \cap \operatorname{Ker} L, x^{T} \Psi(r ; x)<0$, we get

$$
\operatorname{deg}\{H Q N x, \Omega \cap \operatorname{Ker} L, 0\}=\operatorname{deg}\{-x, \Omega \cap \operatorname{Ker} L, 0\} \neq 0 .
$$

Condition ( $b$ ) of Lemma 3.1 is also satisfied. By now we have prove that $\Omega$ satisfies all the requirements in Lemma 3.1. Hence, system (1.2) has at least one $\omega$-periodic solution. The proof is complete.

\section{Global stability of the periodic solution}

In this section, we will obtain sufficient conditions for the global asymptotic stability and global exponential stability of the periodic solution of discrete high-order Hopfield-type networks (1.2). 
Theorem 4.1. Assume that condition (H1), (H2), (H3), and (H4) are satisfied. Furthermore, assume that $\tau_{i j}(n)=\tau_{i j} \in Z^{+}, \sigma_{i j l}(n)=\sigma_{i j l} \in Z^{+}$, and

(H5) There exists a positive real number sequence $\alpha_{i}$ such that

$$
\begin{aligned}
\lambda_{i}= & \alpha_{i}\left(1-e^{\underline{a}_{i}}\right)-L_{i} \sum_{j=1}^{m} \alpha_{j} \theta_{j}(h) b_{j i}^{M}-L_{i} \sum_{j=1}^{m} \alpha_{j} \theta_{j}(h) \tilde{b}_{j i}^{M}-L_{i} \sum_{j=1}^{m} \sum_{l=1}^{m} \alpha_{j} \theta_{j}(h) e_{j i l}^{M} M \\
& -L_{i} \sum_{j=1}^{m} \sum_{l=1}^{m} \alpha_{j} \theta_{j}(h) \widetilde{e}_{j i l}^{M} M-L_{i} \sum_{j=1}^{m} \sum_{l=1}^{m} \alpha_{j} \theta_{j}(h) e_{j l i}^{M} M \\
& -L_{i} \sum_{j=1}^{m} \sum_{l=1}^{m} \alpha_{j} \theta_{j}(h) \widetilde{e}_{j l i}^{M} M>0, \quad i=1,2, \ldots, m .
\end{aligned}
$$

Then the w-periodic solution of (1.2) is unique and all other solutions of (1.2) converges to its unique $\omega$-periodic solutions.

Proof. According to Theorem 3.2, we know that (1.2) has a $\omega$-periodic solution $x^{*}(n)=$ $\left(x_{1}^{*}(n), x_{2}^{*}(n), \ldots, x_{m}^{*}(n)\right)^{T}$. Obviously, if this periodic solution is globally attractivity, then it is unique. Let $x(n)=\left(x_{1}(n), x_{2}(n), \ldots, x_{m}(n)\right)^{T}$ is an arbitrary solution of (1.2) and let

$$
\begin{aligned}
x_{i}(n+1)-x_{i}^{*}(n+1)= & -e^{a_{i}(n) h}\left(x_{i}(n)-x_{i}^{*}(n)\right) \\
& +\theta_{i}(h) \sum_{j=1}^{m} b_{i j}(n)\left(f_{j}\left(x_{j}(n)\right)-f_{j}\left(x_{j}^{*}(n)\right)\right) \\
& +\theta_{i}(h) \sum_{j=1}^{m} \tilde{b}_{i j}(n)\left(f_{j}\left(x_{j}\left(n-\tau_{i j}\right)\right)-f_{j}\left(x_{j}^{*}\left(n-\tau_{i j}\right)\right)\right) \\
& +\theta_{i}(h) \sum_{j=1}^{m} \sum_{l=1}^{m} e_{i j l}(n)\left(f_{j}\left(x_{j}(n)\right) f_{l}\left(x_{l}(n)\right)-f_{j}\left(x_{j}^{*}(n)\right) f_{l}\left(x_{l}^{*}(n)\right)\right) \\
& +\theta_{i}(h) \sum_{j=1}^{m} \sum_{l=1}^{m} \tilde{e}_{i j l}(n)\left(f_{j}\left(x_{j}\left(n-\sigma_{i j l}\right)\right) f_{l}\left(x_{l}\left(n-\sigma_{i j l}\right)\right)\right. \\
& \left.+f_{j}\left(x_{j}^{*}\left(n-\sigma_{i j l}\right)\right) f_{l}\left(x_{l}^{*}\left(n-\sigma_{i j l}\right)\right)\right), \quad i=1,2, \ldots, m .
\end{aligned}
$$

Hence,

$$
\begin{aligned}
\left|x_{i}(n+1)-x_{i}^{*}(n+1)\right| \\
\leq-e^{\underline{a}_{i}}\left|x_{i}(n)-x_{i}^{*}(n)\right| \\
\quad+\theta_{i}(h) \sum_{j=1}^{m} b_{i j}^{M} L_{j}\left|x_{j}(n)-x_{j}^{*}(n)\right| \\
\quad+\theta_{i}(h) \sum_{j=1}^{m} \tilde{b}_{i j}^{M} L_{j}\left|x_{j}\left(n-\tau_{i j}\right)-x_{j}^{*}\left(n-\tau_{i j}\right)\right|
\end{aligned}
$$




$$
\begin{aligned}
& +\theta_{i}(h) \sum_{j=1}^{m} \sum_{l=1}^{m} e_{i j l}^{M} M\left(L_{j}\left|x_{j}(n)-x_{j}^{*}(n)\right|+L_{l}\left|x_{l}(n)-x_{l}^{*}(n)\right|\right) \\
& +\theta_{i}(h) \sum_{j=1}^{m} \sum_{l=1}^{m} \tilde{e}_{i j l}^{M} M\left(L_{j}\left|x_{j}\left(n-\sigma_{i j l}\right)-x_{j}^{*}\left(n-\sigma_{i j l}\right)\right|\right. \\
& \left.\quad+L_{l}\left|x_{l}\left(n-\sigma_{i j l}\right)-x_{l}^{*}\left(n-\sigma_{i j l}\right)\right|\right), \quad i=1,2, \ldots, m .
\end{aligned}
$$

Define a Lyapunov functional $V(\cdot)$ by

$$
\begin{aligned}
V(n)=\sum_{i=1}^{m}( & \alpha_{i}\left|x_{i}(n)-x_{i}^{*}(n)\right| \\
& +\alpha_{i} \theta_{i}(h) \sum_{j=1}^{m} \tilde{b}_{i j}^{M} L_{j} \sum_{k=n}^{n+\tau_{i j}-1}\left|x_{j}\left(k-\tau_{i j}\right)-x_{j}^{*}\left(k-\tau_{i j}\right)\right| \\
& +\alpha_{i} \theta_{i}(h) \sum_{j=1}^{m} \sum_{l=1}^{m} \tilde{e}_{i j l}^{M} M\left(L_{j} \sum_{k=n}^{n+\sigma_{i j l}-1}\left|x_{j}\left(k-\tau_{i j}\right)-x_{j}^{*}\left(k-\tau_{i j}\right)\right|\right. \\
& \left.\left.+L_{l}^{n+\sigma_{i j l}-1}\left|x_{k=n}\left(k-\tau_{i j}\right)-x_{l}^{*}\left(k-\tau_{i j}\right)\right|\right)\right) .
\end{aligned}
$$

Then

$$
\begin{aligned}
& \Delta V(n) \leq \sum_{i=1}^{m}( \alpha_{i}\left(e^{\underline{a}_{i}}-1\right)\left|x_{i}(n)-x_{i}^{*}(n)\right|+\alpha_{i} \theta_{i}(h) \sum_{j=1}^{m} b_{i j}^{M} L_{j}\left|x_{j}(n)-x_{j}^{*}(n)\right| \\
&+\alpha_{i} \theta_{i}(h) \sum_{j=1}^{m} \tilde{b}_{i j}^{M} L_{j}\left|x_{j}(n)-x_{j}^{*}(n)\right| \\
&+\alpha_{i} \theta_{i}(h) \sum_{j=1}^{m} \sum_{l=1}^{m} e_{i j l}^{M} M\left(L_{j}\left|x_{j}(n)-x_{j}^{*}(n)\right|+L_{l}\left|x_{l}(n)-x_{l}^{*}(n)\right|\right) \\
&\left.+\alpha_{i} \theta_{i}(h) \sum_{j=1}^{m} \sum_{l=1}^{m} \tilde{e}_{i j l}^{M} M\left(L_{j}\left|x_{j}(n)-x_{j}^{*}(n)\right|+L_{l}\left|x_{l}(n)-x_{l}^{*}(n)\right|\right)\right) \\
& \leq \sum_{i=1}^{m}\left(\alpha _ { i } \left(e^{\left.\underline{a_{i}}-1\right)}+L_{i} \sum_{j=1}^{m} \alpha_{j} \theta_{j}(h) b_{j i}^{M}+L_{i} \sum_{j=1}^{m} \alpha_{j} \theta_{j}(h) \tilde{b}_{j i}^{M}\right.\right. \\
&+L_{i} \sum_{j=1}^{m} \sum_{l=1}^{m} \alpha_{j} \theta_{j}(h) e_{j i l}^{M} M+L_{i} \sum_{j=1}^{m} \sum_{l=1}^{m} \alpha_{j} \theta_{j}(h) \tilde{e}_{j i l}^{M} M \\
&\left.+L_{i} \sum_{j=1}^{m} \sum_{l=1}^{m} \alpha_{j} \theta_{j}(h) e_{j l i}^{M} M+L_{i} \sum_{j=1}^{m} \sum_{l=1}^{m} \alpha_{j} \theta_{j}(h) \tilde{e}_{j l i}^{M} M\right)\left|x_{i}(n)-x_{i}^{*}(n)\right| \\
& \leq-\sum_{i=1}^{m} \lambda_{i}\left|x_{i}(n)-x_{i}^{*}(n)\right| \leq 0 .
\end{aligned}
$$


Summing both sides of (4.5) from 0 to $n-1$, we get

$$
V(n)+\sum_{k=0}^{n-1}\left(\sum_{i=1}^{m} \lambda_{i}\left|x_{i}(k)-x_{i}^{*}(k)\right|\right) \leq V(0),
$$

which yields

$$
\sum_{k=0}^{\infty} \sum_{i=1}^{m} \lambda_{i}\left|x_{i}(k)-x_{i}^{*}(k)\right| \leq V(0)<\infty .
$$

Therefore, we have

$$
\lim _{n \rightarrow \infty}\left(x_{i}(n)-x_{i}^{*}(n)\right)=0
$$

and we can conclude that the $\omega$-periodic solution of (1.2) is globally attractivity and this completes the proof of the theorem.

Next, we will study global exponential stability of the periodic solution of discrete high-order Hopfield-type networks (1.2).

Theorem 4.2. Assume that condition (H1), (H2), (H3), and (H4) are satisfied. Furthermore, assume that $\tau_{i j}(n)=\tau_{i j} \in Z^{+}, \sigma_{i j l}(n)=\sigma_{i j l} \in Z^{+}$, and

(H5)

$$
\begin{gathered}
\underline{a}_{i}>L_{i} \sum_{j=1}^{m} b_{j i}^{M}+L_{i} \sum_{j=1}^{m} \tilde{b}_{j i}^{M}+L_{i} \sum_{j=1}^{m} \sum_{l=1}^{m} e_{j i l}^{M} M L_{i}+L_{i} \sum_{j=1}^{m} \sum_{l=1}^{m} e_{l j i}^{M} M \\
+L_{i} \sum_{j=1}^{m} \sum_{l=1}^{m} \tilde{e}_{j i l}^{M} M+L_{i} \sum_{j=1}^{m} \sum_{l=1}^{m} \tilde{e}_{l j i}^{M} M, \quad i=1,2, \ldots, m .
\end{gathered}
$$

Then the $\omega$-periodic solution of (1.2) is unique and is globally exponentially stable.

Proof. According to Theorem 3.2, we know that (1.2) has a $\omega$-periodic solution $x^{*}(n)=$ $\left(x_{1}^{*}(n), x_{2}^{*}(n), \ldots, x_{m}^{*}(n)\right)^{T}$. Let $x(n)=\left(x_{1}(n), x_{2}(n), \ldots, x_{m}(n)\right)^{T}$ is an arbitrary solution of (1.2), then

$$
\begin{aligned}
& \left|x_{i}(n+1)-x_{i}^{*}(n+1)\right| \\
& \leq-e^{\underline{a}_{i}}\left|x_{i}(n)-x_{i}^{*}(n)\right|+\theta_{i}(h) \sum_{j=1}^{m} b_{i j}^{M} L_{j}\left|x_{j}(n)-x_{j}^{*}(n)\right| \\
& \quad+\theta_{i}(h) \sum_{j=1}^{m} \tilde{b}_{i j}^{M} L_{j}\left|x_{j}\left(n-\tau_{i j}\right)-x_{j}^{*}\left(n-\tau_{i j}\right)\right|
\end{aligned}
$$




$$
\begin{aligned}
& +\theta_{i}(h) \sum_{j=1}^{m} \sum_{l=1}^{m} e_{i j l}^{M} M\left(L_{j}\left|x_{j}(n)-x_{j}^{*}(n)\right|+L_{l}\left|x_{l}(n)-x_{l}^{*}(n)\right|\right) \\
& +\theta_{i}(h) \sum_{j=1}^{m} \sum_{l=1}^{m} \tilde{e}_{i j l}^{M} M\left(L_{j}\left|x_{j}\left(n-\sigma_{i j l}\right)-x_{j}^{*}\left(n-\sigma_{i j l}\right)\right|\right. \\
& \left.+L_{l}\left|x_{l}\left(n-\sigma_{i j l}\right)-x_{l}^{*}\left(n-\sigma_{i j l}\right)\right|\right), \quad i=, 1,2, \ldots, m .
\end{aligned}
$$

Let $F_{i}(\cdot, \cdot), i \in\{1, \ldots, m\}$ be defined by

$$
\begin{aligned}
F_{i}\left(v_{i}, n\right)= & 1-v_{i} e^{-\underline{a}_{i} h}-L_{i} \theta_{i}(h) v_{i} \sum_{j=1}^{m} b_{j i}^{M}-L_{i} \theta_{i}(h) \sum_{j=1}^{m} \widetilde{b}_{j i}^{M} v_{i}^{\tau_{i j}+1} \\
& -v_{i} L_{i} \theta_{i}(h) \sum_{j=1}^{m} \sum_{l=1}^{m} e_{j i l}^{M}-v_{i} L_{i} \theta_{i}(h) \sum_{j=1}^{m} \sum_{l=1}^{m} e_{l j i}^{M} M \\
& -L_{i} \theta_{i}(h) \sum_{j=1}^{m} \sum_{l=1}^{m} \tilde{e}_{j i l}^{M} M v_{i}^{\sigma_{l j i}+1}-L_{i} \theta_{i}(h) \sum_{j=1}^{m} \sum_{l=1}^{m} \tilde{e}_{l j i}^{M} v_{i}^{\sigma_{l j}+1} M,
\end{aligned}
$$

where $v_{i} \in[1, \infty), n \in I_{\omega}, i \in\{1, \ldots, m\}$. Since

$$
\begin{aligned}
F_{i}(1, n)= & 1-e^{-\underline{a}_{i} h}-L_{i} \theta_{i}(h) \sum_{j=1}^{m} b_{j i}^{M}-L_{i} \theta_{i}(h) \sum_{j=1}^{m} \widetilde{b}_{j i}^{M} \\
& -L_{i} \theta_{i}(h) \sum_{j=1}^{m} \sum_{l=1}^{m} e_{j i l}^{M} M-L_{i} \theta_{i}(h) \sum_{j=1}^{m} \sum_{l=1}^{m} e_{l j i}^{M} M \\
& -L_{i} \theta_{i}(h) \sum_{j=1}^{m} \sum_{l=1}^{m} \tilde{e}_{j i l}^{M} M-L_{i} \theta_{i}(h) \sum_{j=1}^{m} \sum_{l=1}^{m} \tilde{e}_{l j i}^{M} M \\
= & \theta_{i}(h)\left(\underline{a}_{i}-L_{i} \sum_{j=1}^{m} b_{j i}^{M}-L_{i} \sum_{j=1}^{m} \tilde{b}_{j i}^{M}-L_{i} \sum_{j=1}^{m} \sum_{l=1}^{m} e_{j i l}^{M} M L_{i}\right. \\
& \left.\quad-L_{i} \sum_{j=1}^{m} \sum_{l=1}^{m} e_{l j i}^{M} M-L_{i} \sum_{j=1}^{m} \sum_{l=1}^{m} \tilde{e}_{j i l}^{M} M-L_{i} \sum_{j=1}^{m} \sum_{l=1}^{m} \tilde{e}_{l j i}^{M} M\right) \\
\geq & \min _{1 \leq i \leq m}\left(\theta_{i}(h)\right) \gamma>0, \quad i=1,2, \ldots, m,
\end{aligned}
$$

where

$$
\begin{aligned}
\gamma= & \underline{a}_{i}-L_{i} \sum_{j=1}^{m} b_{j i}^{M}-L_{i} \sum_{j=1}^{m} \tilde{b}_{j i}^{M}-L_{i} \sum_{j=1}^{m} \sum_{l=1}^{m} e_{j i l}^{M} M L_{i} \\
& -L_{i} \sum_{j=1}^{m} \sum_{l=1}^{m} e_{l j i}^{M} M-L_{i} \sum_{j=1}^{m} \sum_{l=1}^{m} \tilde{e}_{j i l}^{M} M-L_{i} \sum_{j=1}^{m} \sum_{l=1}^{m} \tilde{e}_{l j i}^{M} M,
\end{aligned}
$$


using the continuity of $F_{i}\left(v_{i}, n\right)$ on $[1, \infty)$ with respect to $v_{i}$ and the fact that $F_{i}\left(v_{i}, n\right) \rightarrow$ $-\infty$ as $v_{i} \rightarrow \infty$ uniformly in $n \in I_{\omega}, i=1,2, \ldots, m$, we see that there exist $v_{i}^{*}(n) \in(1, \infty)$ such that $F_{i}\left(v_{i}^{*}(n), n\right)=0$ for $n \in I_{\omega}, i=1,2, \ldots, m$. By choosing $\lambda=\min \left\{v_{1}^{*}, v_{2}^{*}, \ldots, v_{m}^{*}\right\}$, where $\lambda>1$, we obtain $F_{i}(\lambda, n) \geq 0$ for $n \in I_{\omega}, i=1,2, \ldots, m$, then

$$
\begin{aligned}
\lambda e^{-a_{i}(n) h} & +\theta_{i}(h) L_{i} \lambda \sum_{j=1}^{m} b_{j i}^{M}+\theta_{i}(h) L_{i} \sum_{j=1}^{m} \tilde{b}_{j i}^{M} \lambda^{\tau_{j i}+1} \\
& +\theta_{i}(h) L_{i} \lambda \sum_{j=1}^{m} \sum_{l=1}^{m} e_{j i l}^{M} M L_{i}+\theta_{i}(h) L_{i} \sum_{j=1}^{m} \sum_{l=1}^{m} \tilde{e}_{l j i}^{M} M \lambda^{\sigma_{i j l}+1} \leq 1 .
\end{aligned}
$$

Now let us consider

$$
u_{i}(n)=\lambda^{n} \frac{\left|x_{i}(n)-x_{i}^{*}(n)\right|}{\theta_{i}(h)}, \quad n \in(-\tau, \infty)_{Z}, i=1,2, \ldots, m,
$$

where $\lambda>1$. Then it follows from (1.2) and (4.15) that

$$
\begin{aligned}
u_{i}(n+1) \leq & \lambda e^{-\underline{a}_{i} h} u_{i}(n)+\lambda \sum_{j=1}^{m} b_{i j}^{M} L_{j} \theta_{j}(h) u_{j}(n) \\
& +\sum_{j=1}^{m} \widetilde{b}_{i j}^{M} L_{j} \theta_{j}(h) \lambda^{\tau_{i j}+1} u_{j}\left(n-\tau_{i j}\right) \\
& +\lambda \sum_{j=1}^{m} \sum_{l=1}^{m} e_{i j l}^{M} M\left(L_{j} \theta_{j}(h) u_{j}(n)+L_{l} \theta_{l}(h) u_{l}(n)\right) \\
& +\sum_{j=1}^{m} \sum_{l=1}^{m} \widetilde{e}_{i j l}^{M} M\left(L_{j} \theta_{j}(h) \lambda^{\sigma_{i j l}+1} u_{j}\left(n-\sigma_{i j l}\right)+L_{l} \theta_{l}(h) \lambda^{\sigma_{i j l}+1} u_{l}\left(n-\sigma_{i j l}\right)\right) .
\end{aligned}
$$

Define a Lyapunov functional $V(\cdot)$ by

$$
\begin{aligned}
V(n)=\sum_{i=1}^{m}( & u_{i}(n)+\sum_{j=1}^{m} \tilde{b}_{i j}^{M} L_{j} \theta_{j}(h) \lambda^{\tau_{i j}+1} \sum_{s=n-\tau_{i j}}^{n-1} u_{j}(s) \\
& \left.+\sum_{j=1}^{m} \sum_{l=1}^{m} \widetilde{e}_{i j l}^{M} \theta_{j}(h) \lambda^{\sigma_{i j l}+1} M\left(L_{j} \sum_{s=n-\sigma_{i j l}}^{n-1} u_{j}(s)+L_{l} \sum_{s=n-\sigma_{i j l}}^{n-1} u_{l}(s) d s\right)\right)
\end{aligned}
$$


then calculating the $\Delta V(n)=V(n+1)-V(n)$ along (4.16), we have

$\Delta V(n)$

$$
\begin{aligned}
=\sum_{i=1}^{m}( & u_{i}(n+1)-u_{i}(n)+\sum_{j=1}^{m} \widetilde{b}_{i j}^{M} L_{j} \theta_{j}(h) \lambda^{\tau_{i j}+1}\left(u_{j}(n)-u_{j}\left(n-\tau_{i j}\right)\right) \\
& \left.\times \sum_{j=1}^{m} \sum_{l=1}^{m} \tilde{e}_{i j l}^{M} \theta_{j}(h) \lambda^{\sigma_{i j l}+1} M\left(L_{j}\left(u_{j}(n)-u_{j}\left(n-\sigma_{i j l}\right)\right)+L_{l}\left(u_{l}(n)-u_{l}\left(n-\sigma_{i j l}\right)\right)\right)\right) \\
\leq \sum_{i=1}^{m}( & \left(\lambda e^{-\underline{a}_{i} h}-1\right) u_{i}(n)+\lambda \sum_{j=1}^{m} b_{i j}^{M} L_{j} \theta_{j}(h) u_{j}(n)+\sum_{j=1}^{m} \widetilde{b}_{i j}^{M} L_{j} \theta_{j}(h) \lambda^{\tau_{i j}+1} u_{j}(n) \\
& +\lambda \sum_{j=1}^{m} \sum_{l=1}^{m} e_{i j l}^{M} M\left(L_{j} \theta_{j}(h) u_{j}(n)+L_{l} \theta_{l}(h) u_{l}(n)\right) \\
& \left.+\sum_{j=1}^{m} \sum_{l=1}^{m} \tilde{e}_{i j l}^{M} \lambda^{\sigma_{i j l}+1} M\left(L_{j} \theta_{j}(h) u_{j}(n)+L_{l} \theta_{l}(h) u_{l}(n)\right)\right) \\
m & \left(1-\lambda e^{-\underline{a}_{i} h}-L_{i} \theta_{i}(h) \lambda \sum_{j=1}^{m} b_{j i}^{M}-L_{i} \theta_{i}(h) \sum_{j=1}^{m} \tilde{b}_{j i}^{M} \lambda^{\tau_{j i}+1}\right. \\
\leq & \quad-L_{i} \theta_{i}(h) \sum_{j=1}^{m} \sum_{l=1}^{m} e_{j i l}^{M} M-L_{i} \theta_{i}(h) \sum_{j=1}^{m} \sum_{l=1}^{m} e_{l j i}^{M} M \\
& \left.-L_{i} \theta_{i}(h) \sum_{j=1}^{m} \sum_{l=1}^{m} \tilde{e}_{j i l}^{M} \lambda^{\sigma_{j i l}+1} M-L_{i} \theta_{i}(h) \sum_{j=1}^{m} \sum_{l=1}^{m} \tilde{e}_{l j i}^{M} \lambda^{\sigma_{l j i}+1} M\right) u_{i}(n) \leq 0, \quad t>0
\end{aligned}
$$

and hence from (4.17) we have

$$
\sum_{i=1}^{m} u_{i}(n) \leq V(n) \leq V(0), \quad \text { for } n \in Z^{+}
$$

Thus

$$
\begin{aligned}
\sum_{i=1}^{m} u_{i}(n)= & \lambda^{n} \sum_{i=1}^{m} \frac{\left|x_{i}(n)-x_{i}^{*}(n)\right|}{\theta_{i}(h)} \\
\leq & \sum_{i=1}^{m}\left(u_{i}(0)+\sum_{j=1}^{m} \tilde{b}_{i j}^{M} L_{j} \theta_{j}(h) \lambda^{\tau_{i j}+1} \sum_{s=-\tau_{i j}}^{-1} u_{j}(s)\right. \\
& \left.+\sum_{j=1}^{m} \sum_{l=1}^{m} \tilde{e}_{i j l}^{M} \theta_{j}(h) \lambda^{\sigma_{i j l}+1} M\left(L_{j} \sum_{s=-\sigma_{i j l}}^{-1} u_{j}(s)+L_{l} \sum_{s=-\sigma_{i j l}}^{-1} u_{l}(s) d s\right)\right)
\end{aligned}
$$




$$
\begin{aligned}
& \leq \sum_{i=1}^{m}\left(1+L_{i} \theta_{i}(h) \sum_{j=1}^{m} b_{j i}^{M} \lambda^{\tau_{j i}+1} \tau_{j i}+L_{i} \theta_{i}(h) \sum_{j=1}^{m} \sum_{l=1}^{m} e_{i j l}^{M} \lambda^{\sigma_{i j l}+1} M \sigma_{j i l}\right. \\
& \left.\quad+L_{i} \theta_{i}(h) \sum_{j=1}^{m} \sum_{l=1}^{m} e_{l j i}^{M} \lambda^{\sigma_{l j i}+1} M \sigma_{l j i}\right) \sup _{s \in\left[-\tau^{*}, 0\right]}\left|u_{i}(s)-u_{i}^{*}(s)\right| \\
& \leq \Upsilon\left(\sum_{i=1}^{m} \sup _{s \in\left[-\tau^{*}, 0\right]}\left|u_{i}(s)-u_{i}^{*}(s)\right|\right),
\end{aligned}
$$

where

$$
\Upsilon=\max _{1 \leq i \leq m}\left(1+\sum_{j=1}^{m} b_{i j}^{M} L_{j} e^{\epsilon \tau_{i j}} \tau_{i j}+\sum_{j=1}^{m} \sum_{l=1}^{m} e_{i j l}^{M} e^{\epsilon \sigma_{i j l}} M \sigma_{i j l}\left(L_{j}+L_{l}\right)\right) \geq 1,
$$

then

$$
\begin{aligned}
\sum_{i=1}^{m}\left|x_{i}(n)-x_{i}^{*}(n)\right| & \leq \frac{\max _{1 \leq i \leq m}\left(\theta_{i}(h)\right)}{\min _{1 \leq i \leq m}\left(\theta_{i}(h)\right)} \Upsilon\left(\frac{1}{\lambda}\right)^{n}\left(\sum_{i=1}^{m} \sup _{s \in\left[-\tau^{*}, 0\right]}\left|x_{i}(s)-x_{i}^{*}(s)\right|\right) \\
& \leq \delta\left(\frac{1}{\lambda}\right)^{n}\left(\sum_{i=1}^{m} \sup _{s \in\left[-\tau^{*}, 0\right]}\left|x_{i}(s)-x_{i}^{*}(s)\right|\right),
\end{aligned}
$$

where

$$
\delta=\frac{\max _{1 \leq i \leq m}\left(\theta_{i}(h)\right)}{\min _{1 \leq i \leq m}\left(\theta_{i}(h)\right)} \Upsilon \geq 1
$$

and we can conclude that the $\omega$-periodic solution of (1.2) is globally exponentially stable and this completes the proof of the theorem.

Remark 4.3. If we let $e_{i j l}(t)=\tilde{e}_{i j l}(t)=0$, then system (1.2) reduces to the discrete cellular neural networks, and our Theorems 3.2, 4.1, and 4.2 are [6, Theorem 3.1, Theorem 4.1, and Theorem 4.2], respectively. So our results generalized the main results of [6].

\section{Acknowledgment}

This work was supported by the NSF of Gansu Province of China, the NSF of Bureau of Education of Gansu Province of China (0416B-08), the Key Research and Development Program for Outstanding Groups of Lanzhou University of Technology, and the Development Program for Outstanding Young Teachers in Lanzhou University of Technology.

\section{References}

[1] M. Brucoli, L. Carnimeo, and G. Grassi, Associative memory design using discrete-time secondorder neural networks with local interconnections, IEEE Trans. Circuits Systems I Fund. Theory Appl. 44 (1997), no. 2, 153-158.

[2] A. Dembo, O. Farotimi, and T. Kailath, High-order absolutely stable neural networks, IEEE Trans. Circuits and Systems 38 (1991), no. 1, 57-65. 
[3] R. E. Gaines and J. L. Mawhin, Coincidence Degree, and Nonlinear Differential Equations, Lecture Notes in Mathematics, vol. 568, Springer, Berlin, 1977.

[4] E. B. Kosmatopoulos, M. M. Polycarpou, M. A. Christodoulou, and P. A. Ioannou, High-order neural network structures for identification of dynamical systems, IEEE Trans. Neural Networks 6 (1995), no. 2, 422-431.

[5] E. B. Kosmatopoulos and M. A. Christodoulou, Structural properties of gradient recurrent highorder neural networks, IEEE Trans. Circuits Syst. II: Analog Digital Signal Process. 42 (1995), no. 9, 592-603.

[6] Y. Li, Global stability and existence of periodic solutions of discrete delayed cellular neural networks, Phys. Lett. A 333 (2004), no. 1-2, 51-61.

[7] Z. Liu, A. Chen, J. Cao, and L. Huang, Existence and global exponential stability of periodic solution for BAM neural networks with periodic coefficients and time-varying delays, IEEE Trans. Circuits Systems I Fund. Theory Appl. 50 (2003), no. 9, 1162-1173.

[8] Z. Liu and L. Liao, Existence and global exponential stability of periodic solution of cellular neural networks with time-varying delays, J. Math. Anal. Appl. 290 (2004), no. 1, 247-262.

[9] S. Mohamad and K. Gopalsamy, Dynamics of a class of discrete-time neural networks and their continuous-time counterparts, Math. Comput. Simulation 53 (2000), no. 1-2, 1-39.

[10] J. Su, A. Q. Hu, and Z. Y. He, Stability analysis of analogue neural networks, Electron. Lett. 33 (1997), no. 6, 506-507.

[11] Solving a kind of nonlinear programming problems via analog neural networks, Neurocomputing 18 (1998), no. 1-3, 1-9.

[12] H. Xiang, K.-M. Yan, and B.-Y. Wang, Existence and global exponential stability of periodic solution for delayed high-order Hopfield-type neural networks, submitted.

[13] B. Xu, X. Liu, and X. Liao, Global asymptotic stability of high-order Hopfield type neural networks with time delays, Comput. Math. Appl. 45 (2003), no. 10-11, 1729-1737.

[14] T. Zhang, S. S. Ge, and C. C. Hang, Neural-based direct adaptive control for a class of general nonlinear systems, Internat. J. Systems Sci. 28 (1997), no. 10, 1011-1020.

[15] J. Zhou, Z. Liu, and G. Chen, Dynamics of periodic delayed neural networks, Neural Networks 17 (2004), no. 1, 87-101.

Hong Xiang: Institute of Applied Mathematics, Lanzhou University of Technology, Lanzhou, Gansu 730050, China

E-mail address: xiangh1969@sohu.com

Ke-Ming Yan: Institute of Applied Mathematics, Lanzhou University of Technology, Lanzhou, Gansu 730050, China

E-mail address: yankm@lut.cn.

Bai-Yan Wang: Institute of Applied Mathematics, Lanzhou University of Technology, Lanzhou, Gansu 730050, China 


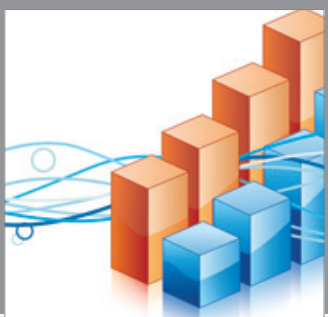

Advances in

Operations Research

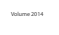

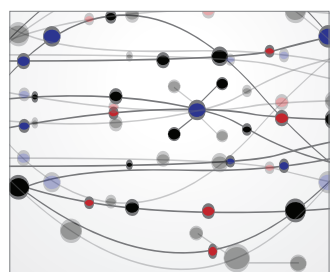

\section{The Scientific} World Journal
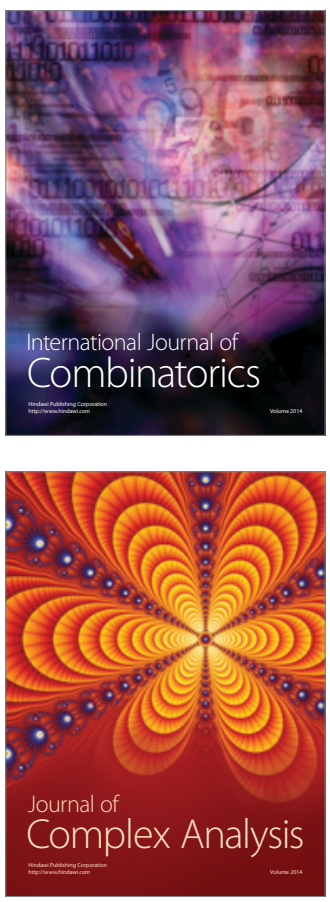

International Journal of

Mathematics and

Mathematical

Sciences
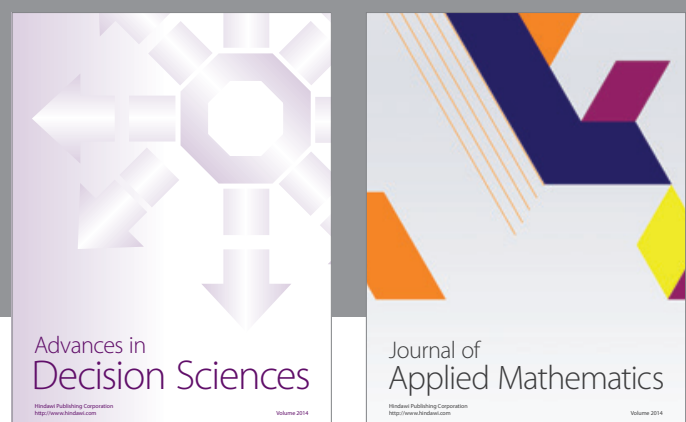

Journal of

Applied Mathematics
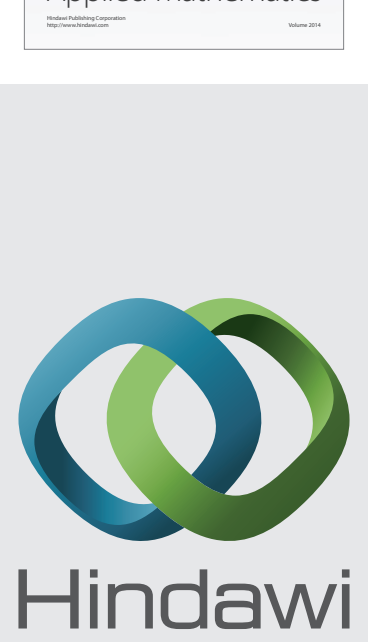

Submit your manuscripts at http://www.hindawi.com
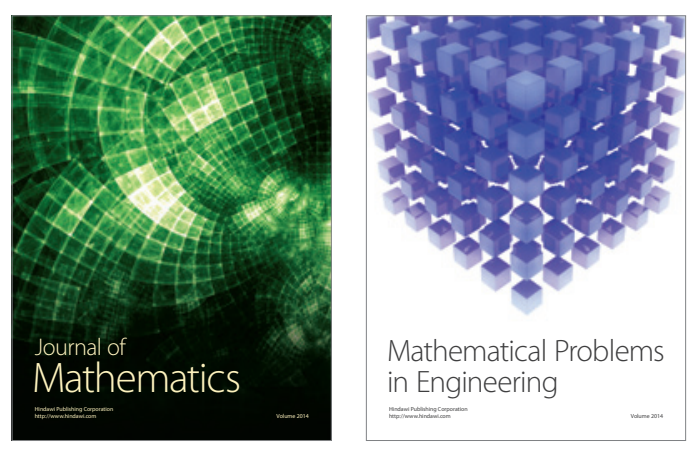

Mathematical Problems in Engineering
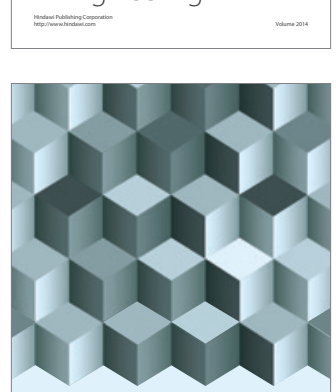

Journal of

Function Spaces
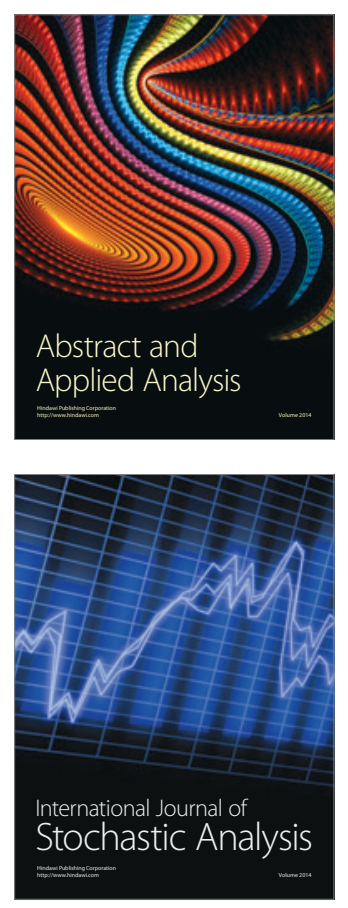

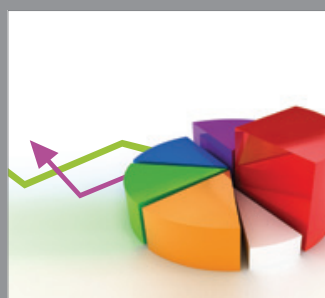

ournal of

Probability and Statistics

Promensencen
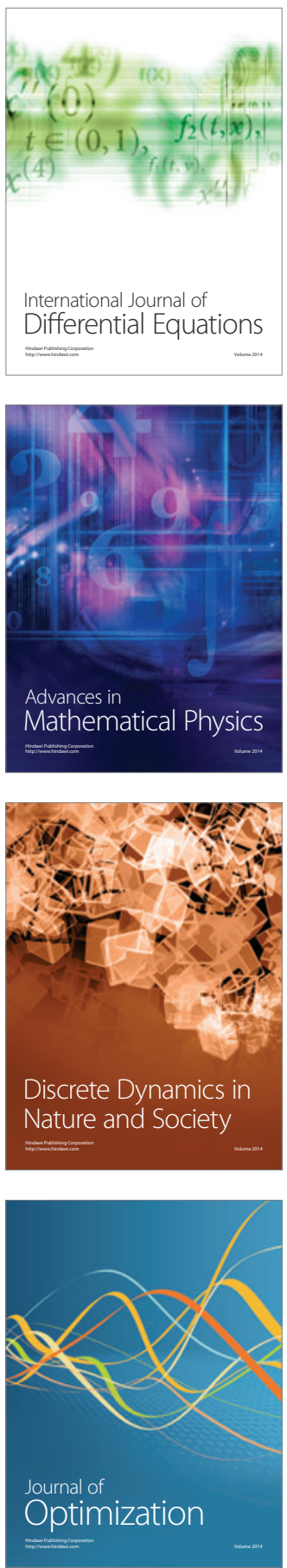